\title{
Healing the Neurophysiological Roots of Trauma: A Controlled Study Examining LORETA Z-Score Neurofeedback and HRV Biofeedback for Chronic PTSD
}

\author{
Ashlie N. Bell ${ }^{*}$, Donald Moss, and Robert J. Kallmeyer \\ Saybrook University, Oakland, California, USA
}

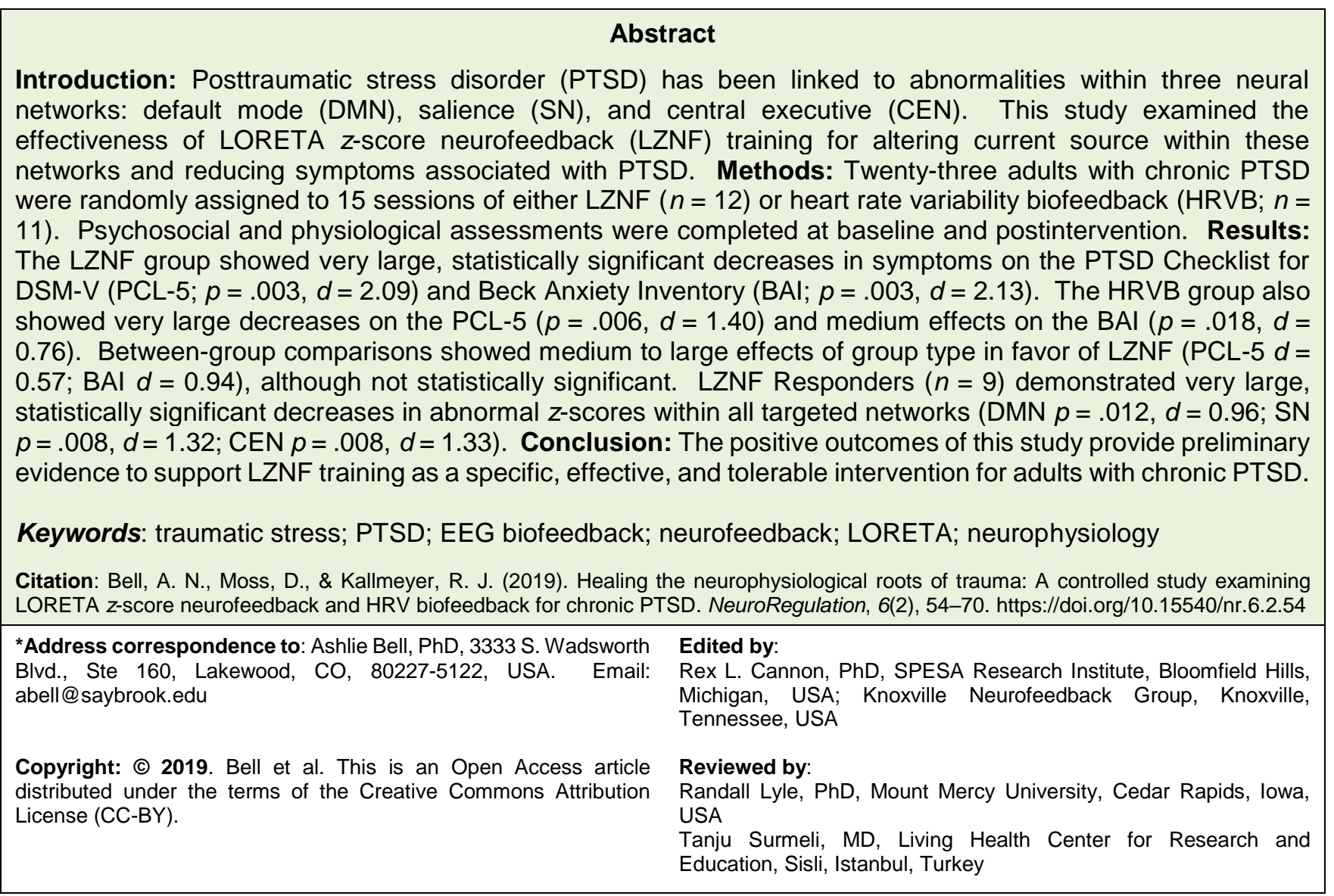

\section{Introduction}

Everything we think, feel, and do is largely governed by a single organ: the human brain. In fact, numerous neuroimaging studies have revealed that alterations in cognition, behavior, mood, and arousal are closely linked to the functional integrity of various brain regions and networks (Bluhm et al., 2009; Daniels et al., 2010; Lanius, Frewen, Tursich, Jetly, \& McKinnon, 2015; Menon, 2011). While decades of research have been dedicated to finding solutions for physical brain injuries and neurodegenerative disorders, less research has examined interventions that target the neurophysiological consequences of something the large majority of humans will face at least once in their lifetime: traumatic stress (Kessler et al., 2017).

\section{Neurophysiological Abnormalities}

Posttraumatic stress disorder (PTSD) has been linked to a number of abnormalities in neural activation patterns, particularly within three intrinsic connectivity networks: the default mode network (DMN), salience network (SN), and central executive network (CEN; Dunkley et al., 2015; Engdahl et al., 2010; Imperatori et al., 2014; Jokić-Begić \& Begić, 2003; Patel, Spreng, Shin, \& Girard, 2012; Todder et al., 2012; van der Kolk, 2006; Wahbeh \& Oken, 2013). 
The $\mathrm{DMN}$ is known for its role in both autobiographical and interpersonal functioning, especially in relation to self-awareness and identity (Menon, 2011). Neuroimaging studies have observed that individuals with PTSD tend to show altered functional connectivity within this network, which might underlie some common experiences reported by trauma survivors, such as relational challenges, depersonalization, and identity alterations (Bluhm et al., 2009; Daniels et al., 2010; Lanius et al., 2015; Patel et al., 2012).

The SN is involved in shifting attention toward or away from internal and external stimuli (Menon, 2011). Individuals with PTSD often show either an overengagement or underengagement of this network, as well as altered connectivity within the network (Lanius et al., 2015). These neurophysiological patterns might contribute to alterations in arousal (e.g., hyperarousal or dissociation), misinterpretation of ambivalent stimuli (e.g., hypervigilance, heightened startle response, etc.), and avoidance (Lanius et al., 2015; Patel et al., 2012; Simmons et al., 2008; Simmons, Strigo, Matthews, Paulus, \& Stein, 2009).

The CEN is known for its role in higher-level cognitive functioning, such as attention, decision-making, planning, working memory, verbal learning, and time perception (Menon, 2011). PTSD has been associated with a failure to properly recruit this network, which might underlie impairments in cognition, difficulty concentrating, and altered time perception during flashbacks (Daniels et al., 2010; Lanius et al., 2015; Patel et al., 2012). The extensive evidence behind such neurophysiological abnormalities provides a strong rationale for interventions that directly target these underlying patterns.

\section{Neurofeedback Training for PTSD}

Neurofeedback training is a psychophysiological intervention designed to alter brain activation patterns toward healthier levels of functioning. This intervention utilizes neuroimaging and a braincomputer interface to read neural activity in real time and feed that information back to clients in the form of audiovisual cues to assist them in self-regulating their brainwave activation patterns (Engelbregt et al., 2016; Lanius et al., 2015). Numerous studies have found neurofeedback to be effective for alleviating symptoms associated with a wide variety of cognitive, emotional, and neurological disorders (Arns et al., 2017; Arns, de Ridder, Strehl, Breteler, \& Coenen, 2009; Panisch \& Hai, 2018; Reiter, Andersen, \& Carlsson, 2016). Moreover, neuroimaging studies have shown alterations in both the functional activation patterns and structural volume of targeted brain regions following neurofeedback training (Ghaziri et al., 2013; Markiewicz, 2017).

An extensive systematic review of the literature by the first author found 10 studies that provided quantifiable data of preintervention to postintervention changes following neurofeedback (Bell, 2018). All studies observed medium to large improvements in PTSD symptoms and/or related neural activity, in accordance with the particular variables measured (Foster \& Thatcher, 2015; Gapen et al., 2016; HuangStorms, Bodenhamer-Davis, Davis, \& Dunn, 2006; Kluetsch et al., 2014; Paret et al., 2014; Peniston \& Kulkosky, 1991; Peniston, Marrinan, Deming, \& Kulkosky, 1993; Pop-Jordanova \& Zorcec, 2004; Ros, Baars, Lanius, \& Vuilleumier, 2014; Smith, 2008; van der Kolk et al., 2016; Walker, 2009). Three of these studies also conducted follow-up assessments, which found improvements to be maintained over an extended period (1-26 months) in most participants. However, most studies utilized convenience samples, and only three included a control or comparison group. Of these, the most recent randomized controlled trial found neurofeedback to produce more significant improvements in affect regulation, identity impairments, abandonment concerns, and overall PTSD symptoms than a treatment-as-usual condition (i.e., psychotherapy and medication; van der Kolk et al., 2016).

The large majority of these studies utilized 20 to 40 sessions of traditional surface electroencephalogram (EEG) neurofeedback. Only one small case series by Foster and Thatcher (2015) utilized a newer modality called low resolution electromagnetic tomography analysis (LORETA) $z$-score neurofeedback. The case series examined 11 veterans with comorbid PTSD and traumatic brain injury, and the number of sessions ranged from 11 to 27 . The authors reported significant $(p<.01)$ changes in LORETA current source density (CSD) within the region of training for all subjects, yielding very large effect sizes (mean $d=$ 1.78) for nine of the veterans and moderate effects (mean $d=0.466$ ) for the other two. These neurophysiological changes were also accompanied by substantial improvements in symptoms. Promising results such as these justify further exploration into the use of this newer neurofeedback modality as an intervention for PTSD and other mental health disorders.

LORETA Z-Score Neurofeedback

LORETA $z$-score neurofeedback (LZNF) is one of the most advanced, comprehensive, and targeted 
modalities of neurofeedback training available. LORETA utilizes a 19-channel EEG cap and threedimensional (3-D) source imaging to determine the specific source of an electric dipole (Pascual-Marqui, Michel, \& Lehmann, 1994). As such, while surface EEG is known to have poor spatial resolutions (i.e., 22-37 $\mathrm{cm}^{3}$ ), the use of LORETA brings these levels down to $7 \mathrm{~mm}^{3}$, all while maintaining the optimal temporal resolutions of EEG within the millisecond time domain (Pascual-Marqui, Esslen, Kochi, \& Lehmann, 2002). Thus, the use of this imaging technology allows for targeted, real-time training of individual brain regions, even deeper within the cortex (Krigbaum \& Wigton, 2014; Thatcher, 2010).

Power and connectivity metrics for each Brodmann area can then be compared against the FDA registered NeuroGuide normative database of agematched, neurotypical individuals (i.e., without neurological or psychological impairments; Thatcher, North, \& Biver, 2005; Thatcher, Walker, Biver, North, \& Curtin, 2003). From this comparison, a $z$-score is derived for each metric, which assumes a normal Gaussian distribution to define the level of deviation from the average of that normative population (Thatcher \& Lubar, 2009). During LZNF training, the $z$-scores for all targeted metrics are computed in real time and trained in the direction of $z=0$. Although limited, research thus far has demonstrated that LZNF produces clinically-meaningful improvements in both symptoms and brain activity for a variety of disorders, including traumatic brain injury (Koberda, 2015a), depression (Koberda et al., 2014b), anxiety (Koberda et al., 2014b; Lambos \& Williams, 2015a), addiction (Cannon, Lubar, Sokhadze, \& Baldwin, 2008), seizures (Frey \& Koberda, 2015; Koberda, 2015b), attention-deficit/hyperactivity disorder (Decker, Roberts, \& Green, 2015; Koberda et al., 2014a), autism (Koberda, 2012), cognitive dysfunction (Koberda, 2014b; Lambos \& Williams, 2015b), and cerebrovascular accident (Koberda, 2014a). These outcomes were produced after an average of 10 to 20 sessions, which is less than the 30 to 40 sessions generally required for traditional neurofeedback.

\section{Method}

\section{Study Design}

The purpose of this study was to assess the effectiveness and specificity of LZNF training, as compared to HRVB training, for reducing mental health symptoms, improving autonomic regulation, and regulating abnormal brainwave activity in adults with chronic PTSD (i.e., symptoms for a minimum of 6 months following a traumatic event).
HRVB as an active control for LZNF. Heart rate variability (HRV) is a measure of beat-to-beat heart rate intervals that is often used as a measure of autonomic regulation (McCraty \& Shaffer, 2015; Thayer, Ahs, Fredrikson, Sollers, \& Wager, 2012). HRV biofeedback (HRVB) training utilizes electrocardiography and a respiratory belt, paired with a breath pacer and audiovisual feedback, to train the heart toward healthier levels of HRV (Thayer et al., 2012). This modality of biofeedback has previously demonstrated effectiveness for reducing PTSD symptoms, even when compared to various control conditions (Ginsberg, Berry, \& Powell, 2010; Reyes, 2014; Tan, Dao, Farmer, Sutherland, \& Gevirtz, 2011; Zucker, Samuelson, Muench, Greenberg, \& Gevirtz, 2009). This qualified HRVB training as an active control condition for the experimental LZNF intervention in this study. It also offered a more ethical option than sham neurofeedback for this sensitive population. The use of HRVB for comparison also provided the opportunity to closely match most of the LZNF training conditions, such as real-time measurement of psychophysiological data, self-regulation training with audiovisual cues, resting time in front of a computer monitor, and interactions with a therapist. To maintain similar levels of placebo and nocebo between the groups, all participants were provided brief psychoeducation around the intervention they would receive and were told that, "as far as we know, both interventions provide equal opportunity for benefit, although neither is guaranteed to benefit."

\section{Eligibility and Enrollment}

Following approval of all aspects of this study by the Saybrook Institutional Review Board, adults between the ages of 18 and 80 were recruited via advertisements on social media, in health and mental health centers, and in various community locations throughout the greater Denver/Boulder area. Eligibility criteria was defined prior to recruitment and all interested individuals completed prescreening. Individuals were eligible if (a) they self-reported having experienced a traumatic event, (b) 6 months or more had passed since the traumatic event, (c) their total score on the PTSD Checklist for DSM-V (PCL-5) was greater than 20, (d) they were proficient in English, and (e) they were between the ages of 18 and 80. Exclusion criteria included (a) moderate to severe brain injury, (b) current diagnosis of a seizure disorder, (c) current diagnosis of a personality disorder, (e) active psychosis, (f) active suicidal ideation, and $(\mathrm{g})$ pregnancy. Participants were also asked to refrain from making changes in their current treatment regimens or engaging in other brainoriented interventions for the duration of this study. 
Eligible participants were officially enrolled in the study upon signing the IRB-approved informed consent form, which spelled out the procedures, risks, and potential benefits of the study.

\section{Measures and Procedures}

This study aimed to examine the effectiveness of LZNF training for reducing PTSD symptoms, improving HRV, and normalizing neural activation patterns associated with PTSD. Seven outcome measures were assessed within approximately one week prior to starting training (Time 1) and one week after the 15th training session (Time 2).

Demographic and presession questionnaires. Prior to initiating training, all participants completed a demographic questionnaire, which assessed for general demographic data (e.g., age, gender, etc.) as well as potential confounding variables (e.g., concurrent practices, medication, etc.). Additionally, participants completed a presession questionnaire at the beginning of each session to track subjective changes in symptoms and assess for factors that could impact physiological measures that day, such as pain, substance use, and sleep quality.

Psychosocial assessment. For each assessment, participants completed two self-report symptom questionnaires: the PTSD Checklist for DSM-V (PCL-5) and the Beck Anxiety Inventory (BAI). The PCL-5 closely correlates with the symptoms outlined in the Diagnostic and Statistical Manual of Mental Disorders (5th ed.; DSM-V; American Psychiatric Association, 2013; Blevins, Weathers, Davis, Witte, \& Domino, 2015) and assesses the frequency and severity of PTSD symptoms using a Likert-type scale. For this study, PTSD symptom scores were computed by summing the total score for all symptoms, producing a continuous score ranging from 0 to 80 . The BAl assesses symptoms of anxiety up to three levels of severity. This questionnaire was utilized to more thoroughly examine changes in psychophysiological anxiety symptoms associated with PTSD. The severity level for each item was attributed a number (i.e., $0-3$ ) and summed for a total anxiety score ranging from 0 to 63 .

Psychophysiological assessment. EEG and HRV data were recorded in the initial and final assessment sessions. A third recording was completed around the midpoint of the intervention to check in on participants' response to training and update training protocols in accordance with changing psychophysiological states. Interim assessments such as this are common, and often necessary, in clinical practice.
HRV recording and artifacting. HRV data was recorded simultaneous with the EEG recording using the NeXus-4 amplifier, an EXG sensor cable, Meditrace disposable electrodes, a respiration sensor, and BioTrace+ software (Mind Media BV, Herten, Netherlands). Electrocardiographic activity was recorded using the forearm placement method, which is a minimally invasive placement that is less susceptible to artifact than placements such as the hand (Shaffer \& Combatalade, 2013). Participants placed the respiration belt around their own waists approximately 2 inches above the navel.

Prior to analyzing this data, visible artifacts were manually removed within the Biotrace+ software. The file was then imported into Kubios 2.1 software (Biosignal Analysis and Medical Imaging Group, Kuopio, Finland) for more detailed artifacting and analysis. For this study, the HRV analyses examined the standard deviation of intervals between normal heart beats (SDNN) and the root mean square of successive differences (RMSSD).

EEG recording and artifacting. Scalp voltages were recorded using a 19-channel electrode cap (Electro-cap International, Inc., Eaton, $\mathrm{OH}$ ) corresponding to the 10-20 international system and referenced to linked ears. Electrode sites were prepared until the impedance level at each site was less than $5 \mathrm{k} \Omega$, and electrical signals were amplified using the Brainmaster Discovery 24E amplifier (BrainMaster Technologies, Inc., Bedford, OH). For each assessment, 10 min of EEG data (i.e., 5 min with eyes open and 5 min with eyes closed) were recorded in an at-rest condition using the NeuroGuide 2.9.1 software (Applied Neuroscience, Inc., Largo, FL).

Each EEG recording was first edited using the automatic artifacting feature in the NeuroGuide 2.9.1 software to remove stereotypical artifacts such as eye blinking and electrode pops. This was followed by a manual scan of the full EEG recording to remove any artifact data that the software had incorrectly selected (e.g., pulse artifact, lateral eye movement, electromyographic activity from temporal or frontal muscles, etc.) and add any true EEG data that it had inaccurately omitted (e.g., abnormal EEG activity). The total selection in each recording included a minimum of 2 minutes of clean EEG data, which is the recommended minimum in accordance with the algorithm of the NeuroGuide database ( $R$. Thatcher, personal communication, August 10, 2017).

Training Protocol Selection and Procedures All participants attended 15 sessions of the training to which they were assigned (i.e., LZNF or HRVB) at a 
rate of two sessions per week. Each session included 20 minutes of direct training, divided into 4 rounds of 5 min. A third-party software, Zukor Media Player (Zukor Interactive, Inc., Las Vegas, NV) was utilized to provide identical audiovisual feedback between the two groups. This feedback was provided in the form of movies, which were chosen by participants from a limited selection. The chosen movie was then connected to the individual's training protocol so that the screen and volume zoomed in and out as participants' physiological measures moved in and out of the set thresholds.

LZNF training protocol. As a way of standardizing an individualized training modality, NeuroGuide's Symptom Checklist-Neural Network Match (SCLFNM) method was utilized to generate each participant's training protocol (Thatcher, 2013). Three networks (i.e., CEN, SN, and DMN) and 5 metrics (i.e., amplitude, coherence, phase, phase shift, and phase lock) were selected for each LZNF participant. The software then automatically compared the LORETA metrics from the client's baseline EEG recording to those of the NeuroGuide normative database, ultimately selecting all metrics within these networks that showed abnormal levels of activity in the individual's brain. The Z-Tunes method was utilized for reward provision, which required two selection criteria to be met for a reward to be received: (1) $70 \%$ of the targeted metrics were within the set $z$-score threshold and (2) the average of the remaining $30 \%$ were moving in the direction of $z=0$ (i.e., a negative slope over time). The $z$-score threshold was manually adjusted throughout each session to maintain a reward rate of 24 to 36 rewards per minute (i.e., 40\%-60\%). When participants successfully achieved a $50 \%-60 \%$ reward rate, the $z$-score threshold was decreased by 1 or 2 tenths of a $z$-score for the next 5-min round (e.g., $z=2.9$ to $z=$ 2.8). In the operant conditioning paradigm, this process has been termed shaping and refers to the reinforcement of successive approximations toward a targeted outcome (Strehl, 2014). Thus, the video feedback in this study guided participants to systematically lower $z$-scores for the trained brain regions, promoting a gradual normalization of the three neural networks.

HRVB training protocol. The HRVB protocol for this study was largely based on the resonant frequency training protocol outlined by Lehrer, Vaschillo, and Vaschillo (2000). This is one of the most common protocols in HRVB training and has previously demonstrated effectiveness for reducing PTSD symptoms (Tan et al., 2011). Each participant's resonant frequency was determined through assessment of five different breathing rates and analyzed for the best convergence of features (e.g., phase relations between heart rate and breathing, peak-trough amplitude, etc.). A breath pacer was then matched to the rate of each participant's resonant frequency and added to the training screen. The combination of breath pacer and video feedback guided participants to breathe at the resonant frequency and increase HRV metrics.

\section{Data Analysis}

The data for this study was analyzed using SPSS software, version 25 . For each outcome measure, the difference from Time 1 to Time 2 was first measured within each of the groups separately and then assessed for differences between the groups. Due to the small, heterogenous sample of this study, nonparametric, signed rank Wilcoxon and MannWhitney $U$ analyses were utilized in place of paired and independent samples $t$-tests. Statistical significance was set at $\alpha=.050$ for all analyses and SPSS output was a $p$ value. Cohen's d effect sizes were also calculated to assess the magnitude and practical importance of observed changes (Weaver \& Goldberg, 2012). These effect sizes are commonly categorized as small $(d=0.20)$, medium $(d=0.50)$, and large $(d \geq 0.80)$.

LORETA CSD z-scores. The final selection of EEG data from each Eyes Open recording was run through NeuroGuide's LORETA 3-D source analysis, which compares the CSD of each Brodmann area against the LORETA normative database to generate $z$-scores. These $z$-scores were then exported from NeuroGuide and imported into an Excel spreadsheet for further analysis. The center voxel was selected for each frequency and Brodmann area within the targeted neural networks and then separated for individual network analyses. Due to the canceling effects of averaging negative and positive $z$-scores, all $z$-scores were converted to absolute values prior to any further analysis. To counter the dampening effects of averaging a large quantity of $z$-scores (i.e., $600-780 z$-scores per network), the total number of significant (i.e., beyond \pm 1.96 ) $z$-scores was summed for each of the targeted neural networks. Higher numbers reflect higher levels of abnormal brain activity.

LZNF responders. Another challenge inherent to LORETA $z$-score analyses is that, due to significant heterogeneity from participant to participant, as well as differences in baseline levels of absolute and relative power (e.g., overall low power), some participants might show an overall increase in significant $z$-scores while others show a decrease. 
Similar to averaging positive and negative $z$-scores, this can lead to cancelation effects. For this reason, a subgroup of the LZNF group $(n=9)$, termed $L Z N F$ Responders, was analyzed further for each of the neural network analyses. This subgroup consisted of participants in the LZNF group who showed any amount of decrease from Time 1 to Time 2 in the total number of significant CSD $z$-scores within the targeted networks. This provided a more accurate measurement of the size and significance of changes in brainwave activity within the majority $(75 \%)$ of LZNF participants.

\section{Results}

\section{Participant Demographics}

Twenty-four eligible adults were enrolled on a firstcome, first-served basis and alternately assigned between the LZNF group and HRVB group according to the order in which they returned their prescreening materials. Only one participant assigned to the HRVB group withdrew participation prior to completion of the study. Thus, the final sample included 12 participants in the LZNF group and 11 in the HRVB group. Enrolled participants ranged from age 30 to 60 with a mean age of 44 . Of the 23 participants that completed the study, $21(91 \%)$ reported at least one comorbid psychiatric disorder, 18 (78\%) were taking psychiatric medication, and 19 (83\%) were concurrently receiving outside psychotherapeutic support. The LZNF group reported a larger number of comorbid diagnoses at baseline (i.e., LZNF $=21$, HRVB = 15). The latter two variables were similar between the two groups at baseline. Participants engaged in psychotherapy had been attending psychotherapy for several months to several years prior to enrollment in this study, and no participants began psychotherapy during their participation in the study.

All participants endorsed having experienced multiple traumatic events, with a mean of 6 direct experiences per participant reported on the PCL-5 Life Events Checklist. The most commonly endorsed traumatic experiences included sexual trauma $(65 \%)$, physical assault (61\%), childhood abuse or neglect (52\%), lifethreatening illness or injury (48\%), natural disaster $(35 \%)$, work-related trauma (e.g., first responders; $26 \%$ ), and military combat (13\%). Mean baseline measurements were similar between the LZNF and HRVB groups for most variables (see Table 1). The largest baseline differences were in the HRV measures, for which the LZNF group had higher initial levels. There was also substantial variability from participant to participant, particularly in the three network analyses. Such variability is common when examining psychophysiological measurements.

\section{Table 1}

Baseline Mean and Median Measurements for LZNF and HRVB Groups

\begin{tabular}{lllll}
\hline & \multicolumn{2}{c}{ HRVB Group } & \multicolumn{2}{c}{ LZNF Group } \\
\cline { 2 - 5 } & Mean $(S D)$ & Median & Mean $(S D)$ & Median \\
\hline Age & $43.73(8.79)$ & 44.0 & $44.58(13.06)$ & 41.0 \\
PCL-5 Total Score & $49.82(10.16)$ & 47.0 & $46.17(14.23)$ & 45.0 \\
BAI Total Score & $24.91(8.77)$ & 26.0 & $25.50(8.08)$ & 22.5 \\
CEN Total Sig Z-scores & $69.09(71.94)$ & 31.0 & $66.17(99.79)$ & 46.0 \\
SN Total Sig Z-scores & $89.09(82.74)$ & 61.0 & $82.00(119.83)$ & 48.0 \\
DMN Total Sig Z-scores & $74.27(73.82)$ & 51.0 & $70.50(105.04)$ & 39.5 \\
SDNN & $23.59(11.62)$ & 19.5 & $37.82(23.51)$ & 28.80 \\
RMSSD & $22.18(12.13)$ & 18.1 & $34.42(17.82)$ & 29.00 \\
\hline
\end{tabular}

Note. LZNF group $n=12$; HRVB group $n=11 ; S D=$ standard deviation; Sig = significant (i.e., absolute $z>1.96$ ). 


\section{Psychosocial Outcomes}

For the psychosocial measures utilized in this study (i.e., PCL-5 and $\mathrm{BAI}$ ), a decrease in scores represents a decrease in negative symptoms and is thus desirable.

PTSD symptoms. For the HRVB group, total scores on the PCL-5 decreased significantly $(p=.006)$ from Time $1(M=49.82, S D=10.16)$ to Time $2(M=31.18$, $S D=13.53)$ with a very large effect $(d=1.40)$. For the LZNF group, PCL-5 scores also decreased significantly $(p=.003)$ from Time $1(M=46.17, S D=$ 14.23) to Time $2(M=18.08, S D=12.65)$ with a very large size of effect $(d=2.09)$. Considering both groups demonstrated very large, statistically significant improvements, the MWU analysis found the difference between the LZNF and HRVB groups to be statistically nonsignificant $(p=.414)$. The Cohen's $d$ analysis, however, found a medium effect of group type $(d=0.57)$. Figure 1 represents the amount of pre-post change in PCL-5 scores, which was about $40 \%$ greater in the LZNF group compared to the HRVB group.

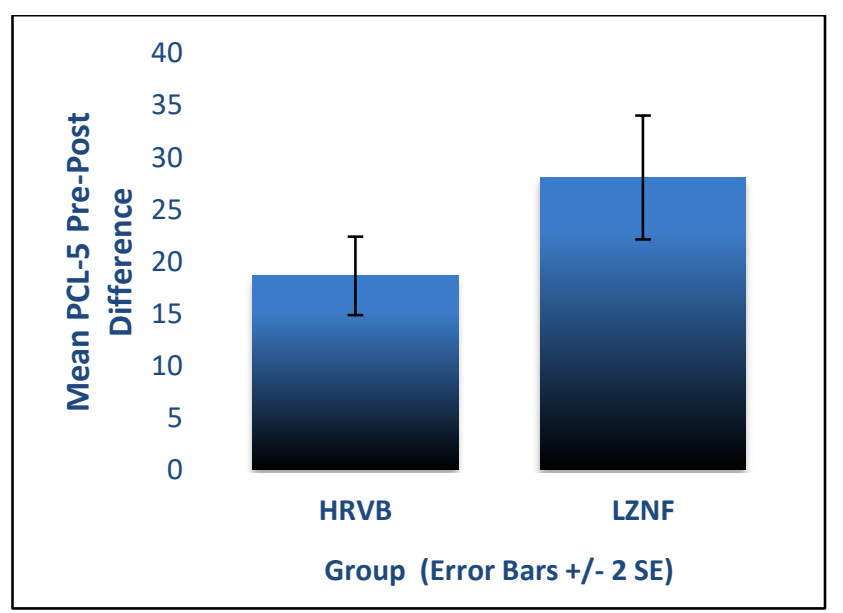

Figure 1. PTSD Checklist for DSM-V pre-post difference in the LZNF and HRVB groups.

Physiological anxiety symptoms. For the HRVB group, total scores on the BAl decreased significantly $(p=.018)$ from Time $1(M=24.91, S D=8.77)$ to Time $2(\mathrm{M}=18.18, \mathrm{SD}=8.9)$ with a medium effect size $(d$ $=0.76$ ). For the LZNF group, BAI scores decreased significantly $(p=.003)$ from Time $1(M=25.50, S D=$ $8.08)$ to Time $2(M=9.83, S D=6.52)$, yielding a very large effect size $(d=2.13)$. Similar to the PCL-5 analyses, MWU results found the BAI difference between the LZNF and HRVB groups to be statistically nonsignificant $(p=.214)$. However, Figure 2 shows that the amount of change from the start to the end of training was about two times larger for the LZNF group than the HRVB group, with a large effect of group type $(d=0.94)$.

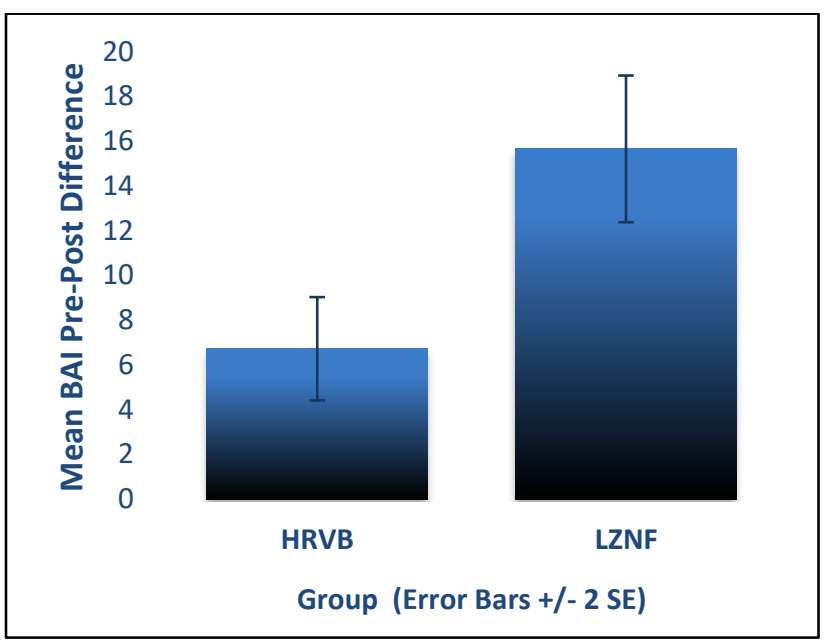

Figure 2. Beck Anxiety Inventory pre-post difference in the LZNF and HRVB groups.

\section{Neural Network Outcomes}

When analyzing changes in neural networks, a decrease in the total number of significant LORETA CSD $z$-scores is desirable and indicates positive change.

Default mode network. The HRVB group showed a slight decrease in the number of significant DMN $z$-scores from Time $1(M=74.27, S D=73.82)$ to Time $2(M=68.27, S D=81.18)$, although this effect was nonsignificant $(p=.790)$ with a negligible size of effect $(d=0.08)$. The LZNF group also showed a decrease in DMN $z$-scores from Time $1(M=70.50, S D=$ 105.04) to Time $2(M=54.33, S D=96.13)$ yielding a small effect $(d=0.27)$, although this difference was also statistically nonsignificant $(p=.213)$. It is important to note this analysis included nine LZNF participants who showed an overall decrease, as well as three LZNF participants who showed an overall increase, thus producing a cancelation of effects. When these canceling effects were removed by analyzing a subgroup of LZNF Responders $(n=9)$ separately, the LZNF training was shown to produce large $(d=0.96)$, statistically significant (Wilcoxon $p=$ .012 ) decreases in the total number of significant DMN z-scores.

The MWU analysis found the DMN difference between the HRVB and full LZNF groups to be statistically nonsignificant $(p=1.00)$ with a small effect of group type $(d=0.17)$. Descriptive analyses, 
however, revealed that the amount of change from preintervention to postintervention in the LZNF group was more than 2.5 times that of the HRVB (see Figure 3 ). For the LZNF Responders, the amount of prepost change was double that of the full LZNF group and over six times that of the HRVB group.

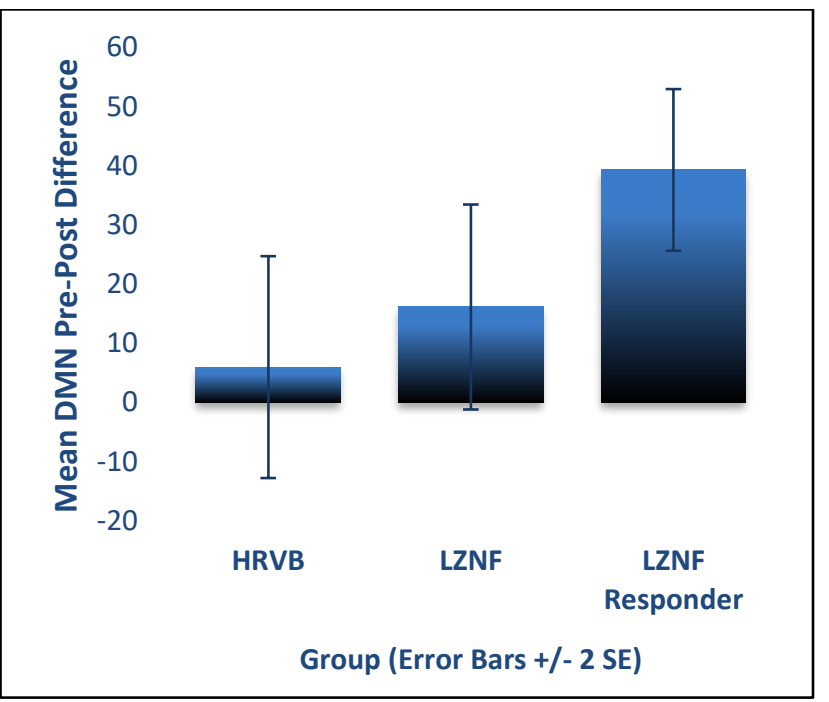

Figure 3. Default mode network pre-post difference in the HRVB, LZNF, and LZNF Responder groups

Salience network. For the HRVB group, there was a modest decrease in the number of significant SN $z$-scores from Time $1(M=89.09, S D=82.74)$ to Time $2(\mathrm{M}=74.91, \mathrm{SD}=115.85)$, although this difference was statistically nonsignificant $(p=.625)$ and yielded a small effect size $(d=0.14)$. The LZNF group also showed a decrease in SN $z$-scores from Time $1(M=$ 82.00, $S D=119.83)$ to Time $2(M=54.42, S D=$ $102.05)$ with a small/medium size of effect $(d=0.49)$, although this difference was also found nonsignificant (Wilcoxon $p=.213$ ). As with the DMN, this analysis included some participants who showed an increase from pretest to posttest. The LZNF Responders subgroup, however, showed very large $(d=1.32)$, statistically significant $(p=.008)$ effects of training from Time $1(M=101.67, S D=133.38)$ to Time $2(M$ $=51.44, S D=117.30)$.

MWU results found the SN difference between the HRVB group and full LZNF group to be statistically nonsignificant $(p=1.00)$ with a very small effect of group type $(d=0.18)$. Figure 4 , however, shows that the amount of pre-post SN change in the LZNF group was about twice that of the HRVB group. The prepost difference in the LZNF Responders was almost twice that of the full LZNF group and over 3.5 times that of the HRVB group.

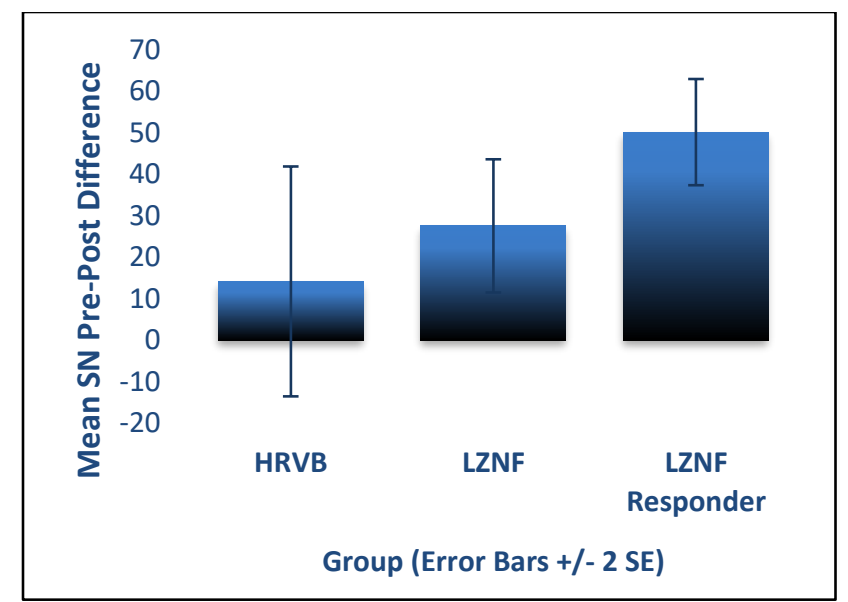

Figure 4. Pre-post SN difference in the HRVB, LZNF, and LZNF Responders groups.

Central executive network. The HRVB group showed a slight pre-post decrease in the number of significant $z$-scores within the CEN from Time $1(M=$ $66.00, S D=69.42)$ to Time $2(M=57.75, S D=94.41)$. This difference was not statistically significant $(p=$ $.790)$ and was very small $(d=0.10)$. The LZNF group showed a larger pre-post change in CEN $z$-scores from Time $1(M=66.17, S D=99.79)$ to Time $2(M=$ $42.50, S D=81.27)$ with a medium effect size $(d=$ 0.59 ), although this difference did not reach statistical significance $(p=.084)$. The LZNF Responders subgroup showed the greatest amount of change from Time $1(M=83.44, S D=110.48)$ to Time $2(M=$ $43.11, S D=95.11)$, with a very large $(d=1.33)$, statistically significant $(p=.008)$ effect of LZNF training.

The MWU analysis found the CEN difference between the HRVB group and LZNF group to be statistically nonsignificant $(p=.414)$ with a small effect of group type $(d=0.29)$. Figure 5 reveals that the amount of change from the start to the end of the intervention was two to three times greater in the LZNF group than the HRVB group. The pre-post difference for the LZNF Responders was 1.5 times that of the full LZNF group and almost 4.5 times the magnitude of the HRVB group. 


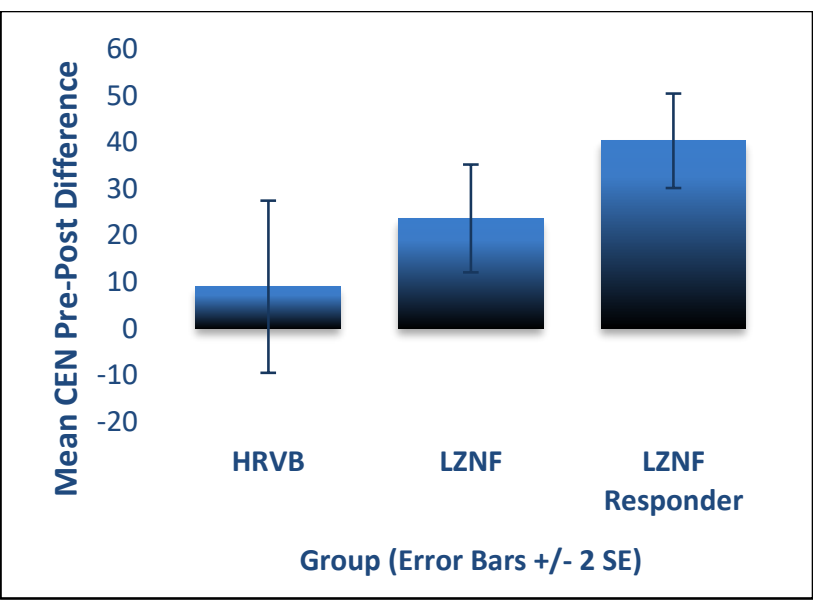

Figure 5. Pre-post CEN difference in the HRVB, LZNF, and LZNF Responders groups.

\section{Heart Rate Variability Outcomes}

When analyzing HRV metrics, an increase in SDNN and RMSSD scores is desirable.

Standard deviation of the NN Interval (SDNN). For the HRVB group, mean SDNN scores increased from Time $1(M=24.90, S D=11.97)$ to Time $2(M=29.82$, $S D=17.73)$ with a small effect size $(d=0.36)$, although this difference was not statistically significant $(p=.182)$. The LZNF group started with a higher mean SDNN and showed a slight decrease $(d$ $=0.11)$ from Time $1(M=37.82, S D=23.51)$ to Time $2(M=36.53, S D=18.32)$, although this change was nonsignificant $(p=.814)$. When comparing the HRVB and LZNF groups, the effect of group type was of medium size $(d=0.58)$, although not statistically significant $(p=.684)$.

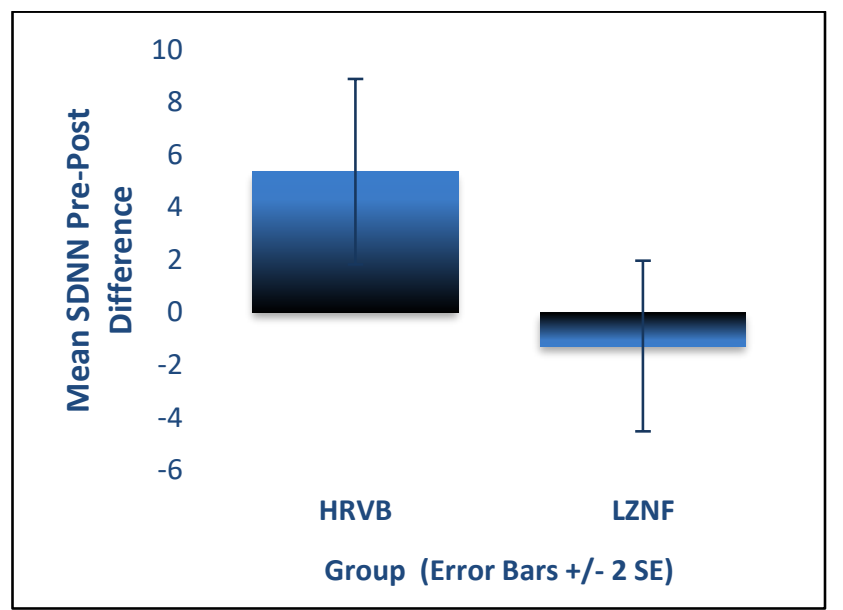

Figure 6. SDNN pre-post difference in the HRVB and LZNF groups.
Root mean square of the successive differences (RMSSD). For the HRVB group, RMSSD means increased from Time $1(M=22.18, S D=12.13)$ to Time $2(M=23.93, S D=16.77)$, although by a very small amount $(d=0.12)$ not reaching statistical significance $(p=.722)$. Similar to the SDNN analyses, the LZNF group showed a slight decrease in RMSSD from Time $1(M=34.42, S D=17.32)$ to Time $2(M=31.51, S D=12.74)$, although this effect was small $(d=0.25)$ and nonsignificant $(p=.530)$. The difference between groups was nonsignificant $(p$ $=1.00)$ with a medium effect of group type $(d=0.50)$.

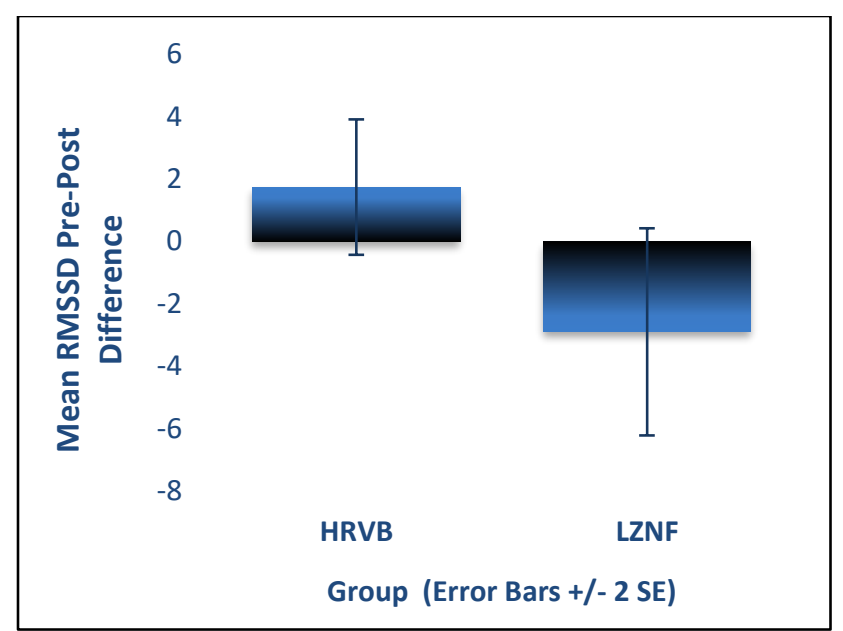

Figure 7. RMSSD pre-post difference in the HRVB and LZNF groups.

\section{Discussion}

This was the first active-controlled study to examine the effectiveness of LZNF training for altering neural activation patterns and alleviating mental health symptoms associated with chronic PTSD. In alignment with prior neurofeedback research, these findings support the notion that neurofeedback is likely an effective intervention for this debilitating condition. Only 15 sessions of LZNF training produced very large, statistically significant effects on both measures of PTSD symptomology. These outcomes were comparable or larger than the active control condition, HRVB, which produced medium to very large decreases in these symptoms. Moreover, these positive outcomes were produced in less than half the number of sessions than the average for traditional neurofeedback modalities (i.e., 30-40 sessions).

The effect sizes for the psychosocial measures in this study compare well with those of common conventional interventions for PTSD, such as eye 
movement desensitization and reprocessing (EMDR), prolonged exposure, cognitive-behavioral therapy, and pharmacotherapy (van der Kolk et al., 2016). Meta-analyses have found the latter three to produce mild to moderate effects in $60 \%$ of participants (Bradley, Greene, Russ, Dutra \& Westen, 2005; Erford et al., 2016; Hoskins et al., 2015; Jonas et al., 2013; Swift \& Greenberg, 2012). Additionally, the rates of completion for the interventions utilized in this study were very high at $100 \%$ for the LZNF group and $92 \%$ for the HRVB group. These retention rates supersede those of psychotherapy and pharmacotherapy, which have been found to vary widely from a maximum average of $80 \%$ to as low as 2\% (Najavits, 2015; Swift \& Greenberg, 2012; Watts et al., 2014). Future research should directly compare LZNF training to these conventional interventions as well as assess any added benefits of an integrated approach.

While many conventional modalities require reexposure to traumatic memories and emotions, LZNF directly targets the underlying neurophysiological patterns without requiring verbal processing. Therefore, LZNF might be especially beneficial for clients who are too hyperaroused, dissociated, or otherwise dysregulated to tolerate processing of traumatic stimuli. Neurofeedback might also be a more appealing, less stigmatizing, and less painful alternative to conventional methods, leading to higher retention rates. Furthermore, many psychotherapists have reported that clients receiving concurrent neurofeedback are better able to self-regulate while processing traumatic content and are thus able to go deeper into the therapeutic process.

Specificity of effects. Although the differences between the LZNF and HRVB groups were not statistically significant, the effects of group type provide preliminary evidence that the effects of each intervention are likely specific to their physiological targets. For example, the LZNF group showed larger effect sizes for both symptom assessments and all three neural networks, with small to large effects of group type; the HRVB group, on the other hand, showed greater improvements in both HRV metrics with medium effects of group type.

\section{Responders vs. Nonresponders}

For the purposes of this study, participants whose $z$-scores moved toward neurotypical levels (i.e., toward $z=0$ ) were considered LZNF Responders, and those whose $z$-scores did not move in this expected direction were termed nonresponders. The rate of responders to nonresponders in this study (i.e., $75 \%$ to $25 \%$ ) was similar to rates observed in other neurofeedback studies (Othmer, 2012). It is worth noting, however, that all three nonresponders in this study did show pre-post changes in their brainwave activity, although this change involved an overall increase in $z$-scores rather than the expected decrease. Of these three participants, two started with low-powered EEGs at baseline and showed an increase in overall power by the end of the study; this might have caused regions and frequencies that were relatively higher to begin with to be boosted over the predefined threshold $(z>1.96)$, resulting in an overall increase in the number of significant $z$-scores. The greatest increases for all participants were within the alpha and beta frequency bands, and two participants showed concurrent decreases in slow wave activity. Slow waves can sometimes indicate neural weakness, so it is possible that a decrease in these slow waves with a concurrent increase in faster frequencies could be representative of decreased neural weakness and increased cortical excitability (R. Thatcher, personal communication, July 29, 2018).

Furthermore, prior research has found alpha brainwaves to be associated with a state of calmness, flow, and mindfulness, and low beta frequencies have been associated with calm, focused attention (Kluetsch et al., 2014; Thompson, Thompson, \& ReidChung, 2015). In fact, a study by Kluetsch et al. (2014) observed a significant increase in alpha power following alpha desynchronization neurofeedback, which was accompanied by feelings of calmness and enhanced functional connectivity within both the DMN and SN. Therefore, an overall increase in these frequencies might not necessarily be undesirable. In line with this thought, the nonresponders in this study reported increases in feelings of calmness, openness, and present moment awareness by the end of the study.

Avoidance and emotional numbness. Nonresponders showed maximal increases in $z$-scores at the midpoint assessment, accompanied by a brief increase in symptoms at some point during the initial stages of training. By the final assessments, however, these $z$-scores had decreased from the midpoint scores (although still higher than baseline) and participants subjectively reported large improvements in symptoms, specifically in relation to memory cohesion, mental clarity, and ability to cope. Notably, all three nonresponders reported a history of significant childhood trauma associated with primary caregivers, as well as prominent symptoms of avoidance and emotional numbing. Two reported extreme difficulty remembering large parts of their traumatic experiences as well. Thus, the initial 
increases in $z$-scores and overall EEG power might be interpreted as a breaking out of avoidance and numbness into more appropriate levels of feeling and processing. Such clients might require additional sessions to first increase overall EEG power and then bring down relatively higher brainwave activity once it becomes more detectable to the training software. Future research should further investigate different subtypes of trauma survivors as they relate to EEG activity and LZNF training.

\section{Limitations, Delimitations, Recommendations for Future Research}

There are a number of variables to consider when interpreting the results of this research. The most significant limitation of this study was the small sample size. Due to the lack of adequate prior research examining LZNF, the available data was insufficient to conduct an accurate power analysis (Leon, Davis, \& Kraemer, 2011). In such cases, authors have proposed the most appropriate sample for this early stage of research should be 10-30 participants per group (Johanson \& Brooks, 2010; Julious, 2005). In line with these recommendations, the final sample in this study was 11-12 participants per group. Nonetheless, a small sample increases the probability of Type II error and might have underpowered this study to achieve statistical significance on outcome measures with lower effect sizes, such as between-group comparisons. A priori sample size calculations using the neural network effect sizes observed in this study determined that, for $80 \%$ statistical power and an alpha level of .05 , the minimum required sample to achieve statistical significance within the LZNF group would have been 94 participants. For the between-group comparisons, a minimum of 188 participants per group would have been necessary. Even so, some outcome measures (i.e., within-group psychosocial assessments and LZNF Responder network changes) produced large enough effects to achieve the hypothesized significance despite the limitations of a small sample.

In the planning of this study, it was understood that it might be underpowered to accurately measure statistical significance; however, considering this was the first controlled study to examine LZNF training as an intervention for PTSD, the purpose of the study was to provide important preliminary data to support future research. For this reason, descriptive statistics and Cohen's d effect sizes were provided for all measures, and nonparametric analyses were utilized in place of inferential statistics for hypothesis testing. Researchers should utilize the methods, outcomes, and lessons learned in this small study to guide the planning of a larger study.
Sample heterogeneity and comorbid diagnoses. Another challenge that arises in both PTSD and psychophysiological research is the significant heterogeneity from subject to subject. The current diagnostic criteria outlined in the DSM-V allows for over 636,000 possible clinical presentations, and different clinical presentations might have different underlying neurophysiological patterns (GalatzerLevy \& Bryant, 2013). Epidemiological surveys have also estimated that about $80 \%$ of adults with PTSD have at least one comorbid mental health disorder and/or substance abuse disorder, which might further diversify neurophysiological patterns in this population (Brady, Killeen, Brewerton, \& Lucerini, 2000; Kessler, Sonnega, Bromet, Hughes, \& Nelson, 1995). To limit the participants in this study to "pure" PTSD (i.e., devoid of comorbidities) would not be fully representative of the PTSD population. Thus, the inclusion criteria for this study was less limiting than some other, more rigorous PTSD studies. Consequently, the resulting sample included a wide diversity of backgrounds, trauma histories, and comorbidities. While such a sample maintains ecological validity, it also presents complications, both for the intervention as well as interpretation of outcomes. Furthermore, the majority of this study's participants reported a history of childhood trauma, which has been found to alter the structural and functional development of the brain (Cassiers et al., 2018). As such, individuals with multiple comorbidities and/or a history of childhood trauma might require additional sessions, as well as a more integrative approach.

In addition to a diversity of trauma histories and symptomology, the participants in this study also showed extensive variability in baseline levels of psychophysiological dysregulation. For example, one participant in the LZNF group started with 113 significant $z$-scores in the CEN while another started with only 8. Participants with higher levels of dysregulation might require more than 15 sessions for complete resolution, while those with lower numbers have little room for statistical improvement (i.e., ceiling effect). Such heterogeneity of the data also leads to a large number of outliers on both ends of the spectrum. The use of nonparametric, signed rank analyses in this study reduced the impact of outliers on hypothesis testing, although the descriptive statistics still reflected this variability. Extensive variability also reduces the statistical power of a study (Dufek, Bates, \& Davis, 1995). Future studies might benefit from predefining eligibility criteria based on a specified range of psychophysiological parameters. A larger sample would also provide the option to cluster participants into different subtypes according 
to symptom presentation, comorbidities, and baseline physiological measures.

Active control vs. sham. This study employed an active comparison group that received HRVB training in place of LZNF training. While this was an appropriate and ethical control condition, findings might have been more robust with the use of a sham (i.e., placebo) control. Sham controls are generally considered the gold standard for assessing the efficacy of an intervention while ruling out the most common confounding variables, such as placebo. However, sham neurofeedback might not be an ethical option for sensitive. Trauma survivors often experience feelings of powerlessness and helplessness, which could be exacerbated by sham conditions in which they are powerless to affect change (Brewin, Andrews, \& Rose, 2000). Moreover, there is some evidence to suggest that sham feedback might reduce a person's ability to effectively learn from future neurofeedback training (Kluetsch et al., 2014; van Boxtel et al., 2012). The World Medical Association's Declaration of Helsinki, which outlines ethical principles for medical research with human participants, recommends that when another effective intervention exists for a particular ailment, an experimental intervention should be tested against that intervention rather than placebo due to the potential for harm related to nontreatment or delayed intervention (Carlson, Boyd, \& Webb, 2004; World Medical Association, 2013).

For these reasons, HRVB was determined to be an ethical control condition while maintaining scientific rigor and controlling for many of the same confounding variables as sham neurofeedback. However, participants were unblinded to the training they were undergoing, which is a complication inherent to the use of active controls. Future research comparing these two conditions might effectively blind participants to the condition by connecting them to both EEG and HRV apparatuses for all training sessions; in this way, all participants would receive true psychophysiological feedback without knowing whether or not that feedback was based on brain activity or heart activity. This would allow for at least a single-blinded condition while still providing the opportunity for all participants to benefit.

Another challenge in comparing an experimental intervention against an active control is that an active control is, by definition, an effective intervention for the target population. This requires experimental conditions to achieve an even larger effect in order to produce statistically significant differences in between-group comparisons. On the other hand, equivalency of outcomes between an experimental condition and another proven intervention actually demonstrates effectiveness in and of itself. In fact, guidelines for evaluating the clinical efficacy of psychophysiological interventions state that, for an experimental intervention to be considered "efficacious," it must be at least "equivalent to a treatment of established efficacy in a study with sufficient power to detect moderate differences" (La Vaque et al., 2002). Although the statistical power of this study was insufficient to declare efficacy, the fact that the LZNF condition was at least equivalent to the HRVB condition makes it a promising alternative for the treatment of chronic PTSD.

\section{Challenges in statistical analysis of LORETA} $z$-scores. The neural analyses in this study calculated the total number of significant, absolute $z$-scores, which resolved many challenges inherent to LORETA $z$-score analyses, such as negativepositive $z$-score cancelations, minor shifts within neurotypical ranges, and dampening effects of averaging multiple $z$-scores. Nonetheless, this method still presented some complications of its own. For example, measuring the CSD for all Brodmann areas and frequencies within each network resulted in a very large number of metrics per network, which increased the extent of intersubject variability and reduced statistical power. Additionally, grouping all frequencies together in a single analysis did not allow for separate examination of changes within each frequency band, thus reducing the ability to adequately assess compensatory dynamics.

However, it would be challenging to find a single brain region and frequency band that would be dysregulated within all participants, especially considering the extensive heterogeneity of the PTSD population. The examination of entire networks in this study increased the chances of encompassing various patterns of dysregulation within a diverse sample, as well as assess changes in overall dysregulation throughout large-scale neural networks. However, limiting LORETA data analyses to a single metric might have provided a less confounded analysis of the size and significance of change, with increased statistical power. Future researchers might consider predetermining a region of interest and creating eligibility criteria based on EEG parameters for that region. As an alternative, future studies could design a reproducible method for selecting a different metric of interest for each participant, such as the region and frequency with the most significant $z$-score. 
This study's method of data analysis also failed to account for individuals starting with low-power EEGs, which might have been responsible for the observed increases in $z$-scores for some of the nonresponders. Although assessment of the LZNF Responders subgroup resolved this issue for the pre-post analyses of that subgroup, all analyses containing the full LZNF group were impacted by these canceling effects. Furthermore, changes were only counted once they surpassed the set cut-off threshold (i.e., 1.96) in either direction. For example, if a $z$-score for $20 \mathrm{~Hz}$ in Brodmann area 13 decreased from 3.4 down to 2.1, it was still above the 1.96 threshold and thus counted as if there was no change (i.e., 1 stays 1 ). On the other hand, if a $z$-score for $10 \mathrm{~Hz}$ in Brodmann area 24 increased by a much smaller amount, such as a shift from 1.94 to 1.97 , it was counted as significant (i.e., 0 becomes 1 ) because it surpassed the predefined threshold. Future research might reduce this complication by measuring changes in the means of all absolute $z$-scores above the 1.96 threshold.

Challenges in statistical analyses of HRV metrics. For the HRV analyses, it is possible that the LZNF group experienced ceiling effects due to high baseline levels of HRV, which were higher than baseline means for the HRVB group. Some participants also showed higher baseline levels of variability due to more chaotic heart rate patterns, such as sudden increases or decreases in heart rate. However, this activity was not in phase with their breathing. Over time, the raw data during training sessions showed improvements in phase relations between heart rate and breathing rate, but this data was not reflected in the predetermined amplitude analyses for this study. Future PTSD research might utilize HRV coherence training in place of amplitude training as well as analyze pre-post changes in coherence.

Additional confounding variables. Within the time frame of this study, many participants were exposed to stressful situations and confounding variables. For example, some participants: (a) experienced significant losses or conflictual periods in their relationships, (b) were re-exposed to trauma-related triggers or people, (c) endured a physical illness or injury, (d) had changes in their job status, (e) were undergoing final examinations in school, (f) were experiencing changes related to seasonal shifts (i.e., fall to winter), and (g) experienced additional stress related to holiday events. Within a naturalistic setting, it is difficult to avoid exposure to such confounding variables. Since one of the key characteristics of PTSD is being triggered by a variety of stimuli, it would not have been appropriate to remove all triggered participants from analysis. However, such events might have had an impact on both training effectiveness and assessments. Future research might explore ways to reduce these variables. Importantly, many participants who were exposed to triggers and stressors reported feeling lower levels of stress reactivity and enhanced levels of selfregulation in response to these stressors than they had prior to the study.

Optimal training targets and thresholds. Future research is needed to further parse out the effectiveness of various neurofeedback training modalities. Research should also assess for optimal reward rates, the optimal number of metrics to be trained simultaneously, and differences in effectiveness and tolerability between the Z-Tunes and All-or-None methods. Differences in audiovisual feedback should also be assessed, taking into account variables such as the effectiveness of direct feedback versus more obscure feedback, as well as levels of motivation and reward to reinforce a desired activation pattern. For example, while bar graphs might provide more direct feedback, movies might be more motivating and thus induce a larger dopamine reward.

A more individualized approach. In order to maintain reproducibility, the training in this study was confined to brain regions within the three predefined networks. However, many participants, including the three nonresponders, showed additional dysregulated brainwave activity outside these networks-sometimes to a greater extent than dysregulation within the networks. It is possible that dysregulated activation patterns of untrained regions might have negatively impacted the training process, especially if overactivation of the trained regions was a compensatory mechanism for dysregulation in outside regions. Consequently, a more individualized, comprehensive training program might have produced even more optimal outcomes for the participants in this study. Future research and clinical practice might utilize this study's protocol design as a starting point and add a manualized method for further individualizing protocols to each client's needs. Alternatively, the SCL-FNM method could be used to select regions associated with an individual's primary symptoms rather limiting the training to predefined networks.

Integration of LZNF and HRVB. Considering both interventions demonstrated positive effects, future research might include a third group to assess for added benefits of integrating LZNF training with HRVB training. Although LORETA neuroimaging can 
reach deeper regions of the cortex than surface EEG neurofeedback, it cannot effectively reach deeper subcortical regions such as the hypothalamus. Any LZNF-induced changes in deeper limbic regions are likely related to modulatory feedback loops between cortical and subcortical regions. HRVB might also exhibit effects on these subcortical regions via feedback loops between the peripheral nervous system and subcortical brain regions (Thompson et al., 2015). In fact, this type of "bottom-up" regulation might have contributed to the positive symptom changes reported by participants in the HRVB group. Future research might utilize fMRI neuroimaging to further explore the effects of each intervention on subcortical regions associated with PTSD. Furthermore, the integration of HRVB and LZNF training might prove more effective than either intervention alone by targeting dysregulated activation patterns from both directions.

\section{Conclusion}

Chronic PTSD is a debilitating condition that, despite conventional treatment attempts, continues to impact millions of lives around the world. Neuroimaging studies have found strong associations between symptoms of PTSD and abnormal neurophysiology, particularly within three large-scale neural networks: the DMN, SN, and CEN. Such evidence demonstrates a need for interventions that more directly target these underlying neurophysiological roots, such as LZNF training. This was the first controlled study to assess the effectiveness of LZNF training for alleviating symptoms, improving autonomic regulation, and regulating abnormal brainwave activity in adults with chronic PTSD.

Despite the aforementioned limitations, the findings of this study provide strong preliminary evidence that LZNF training of the DMN, SN, and CEN is highly effective for reducing both PTSD symptoms and physiological anxiety symptoms in adults with chronic PTSD. HRVB training was also largely effective for reducing these symptoms, and the integration of these interventions might produce even greater outcomes. LZNF training produced large to very large, significant effects on all three targeted neural networks within the majority of trainees (i.e., LZNF Responders). These outcomes were produced in less than half the average number of sessions for traditional neurofeedback modalities. Positive outcomes were also observed across a wide diversity of individuals and comorbidities, indicating that LZNF training might be beneficial for a variety of trauma populations and conditions.
Furthermore, both interventions demonstrated very high rates of attendance and completion, suggesting high levels of feasibility and tolerability. These interventions might also offer a more appealing alternative to psychotherapy and medications, especially for professional populations such as military personnel, firefighters, and police officers. All in all, the outcomes of this study provide promising preliminary evidence to support future research with larger sample sizes.

\section{Author Disclosure}

This study was partially funded by mini-grants from the Foundation for Neurofeedback and Neuromodulation Research (FNNR) and the Foundation for Education and Research in Biofeedback and Related Sciences (FERB). The research was also supported by student scholarships from Applied Neuroscience, Inc. for the use of the NeuroGuide software and database, Mind Media for use of a NeXus-4 system and BioTrace+ software, and Zukor Interactive, Inc. for use of the Zukor Media Player. These organizations were not involved in any part of the intervention or data analysis and thus did not influence the outcomes. The authors of this research have no conflicts of interest or financial gains to report.

\section{References}

American Psychiatric Association. (2013). Diagnostic and statistical manual of mental disorders (5th ed.). Arlington, VA: American Psychitatric Publishing.

Arns, M., Batail, J.-M., Bioulac, S., Congedo, M., Daudet, C., Drapier, D., ... NExT group. (2017). Neurofeedback: One of today's techniques in psychiatry? L'Encéphale, 43(2), 135145. https://doi.org/10.1016/j.encep.2016.11.003

Arns, M., de Ridder, S., Strehl, U., Breteler, M., \& Coenen, A. (2009). Efficacy of neurofeedback treatment in ADHD: The effects on inattention, impulsivity and hyperactivity: A metaanalysis. Clinical EEG and Neuroscience, 40(3), 180-189. https://doi.org/10.1177/155005940904000311

Bell, A. N. (2018). Tuning the traumatized brain, mind, and heart: Loreta z-score neurofeedback and HRV biofeedback for chronic PTSD (Doctoral dissertation, Saybrook University). Retrieved from https://search-proquestcom.tcsedsystem.idm.oclc.org/pqdtglobal/docview/21906817 31/abstract/1ACFB298726042D3PQ/1

BioTrace+ [Computer software]. (n.d.). Herten, Netherlands: Mind Media BV.

Blevins, C. A., Weathers, F. W., Davis, M. T., Witte, T. K., \& Domino, J. L. (2015). The Posttraumatic Stress Disorder Checklist for DSM-5 (PCL-5): Development and initial psychometric evaluation. Journal of Traumatic Stress, 28(6), 489-498._https://doi.org/10.1002/jts.22059

Bluhm, R. L., Williamson, P. C., Osuch, E. A., Frewen, P. A., Stevens, T. K., Boksman, K., ... Lanius, R. A. (2009). Alterations in default network connectivity in posttraumatic stress disorder related to early-life trauma. Journal of Psychiatry \& Neuroscience, 34(3), 187-194. https://www.ncbi.nlm.nih.gov/pmc/articles/PMC2674971/ 
Bradley, R., Greene, J., Russ, E., Dutra, L., \& Westen, D. (2005). A multidimensional meta-analysis of psychotherapy for PTSD. The American Journal of Psychiatry, 162(2), 214-227. https://doi.org/10.1176/appi.ajp.162.2.214

Brady, K. T., Killeen, T. K., Brewerton, T., \& Lucerini, S. (2000). Comorbidity of psychiatric disorders and posttraumatic stress disorder. The Journal of Clinical Psychiatry, 61(Suppl. 7), 2232.

BrainMaster Discovery 24E [Apparatus]. Bedford, OH: BrainMaster Technologies, Inc.

Brewin, C. R., Andrews, B., \& Rose, S. (2000). Fear, helplessness, and horror in posttraumatic stress disorder: Investigating DSMIV criterion A2 in victims of violent crime. Journal of Traumatic Stress, 13(3), 499-509. https://doi.org/10.1023 /A:1007741526169

Cannon, R. L., Lubar, J., Sokhadze, E., \& Baldwin, D. R. (2008). LORETA neurofeedback for addiction and the possible neurophysiology of psychological processes influenced: $A$ case study and region of interest analysis of LORETA neurofeedback in right anterior cingulate cortex. Journal of Neurotherapy, 12(4), 227-241. https://doi.org/10.1080 110874200802501948

Carlson, R. V., Boyd, K. M., \& Webb, D. J. (2004). The revision of the Declaration of Helsinki: Past, present and future. British Journal of Clinical Pharmacology, 57(6), 695-713. https://doi.org/10.1111/j.1365-2125.2004.02103.x

Cassiers, L. L. M., Sabbe, B. G. C., Schmaal, L., Veltman, D. J., Penninx, B. W. J. H., \& Van Den Eede, F. (2018). Structural and functional brain abnormalities associated with exposure to different childhood trauma subtypes: A systematic review of neuroimaging findings. Frontiers in Psychiatry, 9. https://doi.org/10.3389/fpsyt.2018.00329

Daniels, J. K., McFarlane, A. C., Bluhm, R. L., Moores, K. A., Clark, C. R., Shaw, M. E., ... Lanius, R. A. (2010). Switching between executive and default mode networks in posttraumatic stress disorder: Alterations in functional connectivity. Journal of Psychiatry \& Neuroscience, 35(4), 258-266. https://doi.org /10.1503/jpn.090010

Decker, S. L., Roberts, A. M., \& Green, J. J. (2015). LORETA neurofeedback in college students with ADHD. In R. W. Thatcher \& D. S. Foster (Eds.), Z score neurofeedback: Clinical applications (pp. 333-352). San Diego, CA: Academic Press. https://doi.org/10.1016/B978-0-12-801291-8.00014-5

Dufek, J. S., Bates, B. T., \& Davis, H. P. (1995). The effect of trial size and variability on statistical power. Medicine \& Science in Sports \& Exercise, 27(2), 288-295. https://doi.org/10.1249 /00005768-199502000-00021

Dunkley, B. T., Sedge, P. A., Doesburg, S. M., Grodecki, R. J., Jetly, R., Shek, P. N., ... Pang, E. W. (2015). Theta, mental flexibility, and post-traumatic stress disorder: Connecting in the parietal cortex. PloS ONE, 10(4), e0123541. https://doi.org /10.1371/journal.pone.0123541

Electro-Cap system [Apparatus]. Eaton, OH: Electro-Cap International, Inc.

Engdahl, B., Leuthold, A. C., Tan, H.-R. M., Lewis, S. M., Winskowski, A. M., Dikel, T. N., \& Georgopoulos, A. P. (2010). Post-traumatic stress disorder: A right temporal lobe syndrome? Journal of Neural Engineering, 7(6), 066005. https://doi.org/10.1088/1741-2560/7/6/066005

Engelbregt, H. J., Keeser, D., van Eijk, L., Suiker, E. M., Eichhorn, D., Karch, S., ... Pogarell, O. (2016). Short and long-term effects of sham-controlled prefrontal EEG-neurofeedback training in healthy subjects. Clinical Neurophysiology, 127(4), 1931-1937. https://doi.org/10.1016/j.clinph.2016.01.004

Erford, B. T., Gunther, C., Duncan, K., Bardhoshi, G., Dummett, B., Kraft, J., ... Ross, M. (2016). Meta-analysis of counseling outcomes for the treatment of posttraumatic stress disorder. Journal of Counseling \& Development, 94(1), 13-30. https://doi.org/10.1002/jcad.12058
Foster, D. S., \& Thatcher, R. W. (2015). Surface and LORETA neurofeedback in the treatment of post-traumatic stress disorder and mild traumatic brain injury. In R. W. Thatcher \& J. F. Lubar (Eds.), Z score neurofeedback: Clinical applications (pp. 59-92). San Diego, CA: Academic Press. https://doi.org/10.1016/B978-0-12-801291-8.00004-2

Frey, L. C., \& Koberda, J. L. (2015). LORETA z-score neurofeedback in patients with medically refractory epilepsy. Journal of Neurology and Neurobiology, 1(1), 1-4. https://doi.org/10.16966/2379-7150.102

Galatzer-Levy, I. R., \& Bryant, R. A. (2013). 636,120 ways to have posttraumatic stress disorder. Perspectives on Psychological Science, 8(6), 651-662. https://doi.org/10.1177 $/ 1745691613504115$

Gapen, M., van der Kolk, B. A., Hamlin, E., Hirshberg, L., Suvak, M., \& Spinazzola, J. (2016). A pilot study of neurofeedback for chronic PTSD. Applied Psychophysiology and Biofeedback, 41(3), 251-261. https://doi.org/10.1007/s10484-015-9326-5

Ghaziri, J., Tucholka, A., Larue, V., Blanchette-Sylvestre, M., Reyburn, G., Gilbert, G., ... Beauregard, M. (2013). Neurofeedback training induces changes in white and gray matter. Clinical EEG and Neuroscience, 44(4), 265-272. https://doi.org/10.1177/1550059413476031

Ginsberg, J. P., Berry, M. E., \& Powell, D. A. (2010). Cardiac coherence and posttraumatic stress disorder in combat veterans. Alternative Therapies in Health and Medicine, 16(4), $52-60$.

Hoskins, M., Pearce, J., Bethell, A., Dankova, L., Barbui, C., Tol, W. A., ... Bisson, J. I. (2015). Pharmacotherapy for posttraumatic stress disorder: Systematic review and metaanalysis. The British Journal of Psychiatry, 206(2), 93-100. https://doi.org/10.1192/bjp.bp.114.148551

Huang-Storms, L., Bodenhamer-Davis, E., Davis, R., \& Dunn, J. (2006). QEEG-guided neurofeedback for children with histories of abuse and neglect: Neurodevelopmental rationale and pilot study. Journal of Neurotherapy, 10(4), 3-16. https://doi.org /10.1300/J184v10n04 02

Imperatori, C., Farina, B., Quintiliani, M. I., Onofri, A., Castelli Gattinara, P., Lepore, M., ... Della Marca, G. (2014). Aberrant EEG functional connectivity and EEG power spectra in resting state post-traumatic stress disorder: A sLORETA study. Biological Psychology, 102, 10-17. https://doi.org/10.1016 /j.biopsycho.2014.07.011

Johanson, G. A., \& Brooks, G. P. (2010). Initial scale development: Sample size for pilot studies. Educational and Psychological Measurement, $\quad 70(3), \quad 394-400 . \quad$ https://doi.org/10.1177 10013164409355692

Jokić-Begić, N., \& Begić, D. (2003). Quantitative electroencephalogram (qEEG) in combat veterans with posttraumatic stress disorder (PTSD). Nordic Journal of Psychiatry, 57(5), 351-355. https://doi.org/ 10.1080/08039480310002688

Jonas, D. E., Cusack, K., Forneris, C. A., Wilkins, T. M., Sonis, J., Middleton, J. C., ... Gaynes, B. N. (2013). Psychological and pharmacological treatments for adults with posttraumatic stress disorder (PTSD). Comparative Effectiveness Review, 92. Rockville, MD: Agency for Healthcare Research and Quality. https://doi.org/10.1037/e553842013-001

Julious, S. A. (2005). Sample size of 12 per group rule of thumb for a pilot study. Pharmaceutical Statistics, 4(4), 287-291. https://doi.org/10.1002/pst.185

Kessler, R. C., Aguilar-Gaxiola, S., Alonso, J., Benjet, C., Bromet, E. J., Cardoso, G., ... Koenen, K. C. (2017). Trauma and PTSD in the WHO world mental health surveys. European Journal of Psychotraumatology, 8(Suppl. 5), 1353383. https://doi.org /10.1080/20008198.2017.1353383

Kessler, R. C., Sonnega, A., Bromet, E., Hughes, M., \& Nelson, C. B. (1995). Posttraumatic stress disorder in the National Comorbidity Survey. Archives of General Psychiatry, 52(12), 1048-1060. /archpsyc.1995.03950240066012 
Kluetsch, R. C., Ros, T., Théberge, J., Frewen, P. A., Calhoun, V. D., Schmahl, C., ... Lanius, R. A. (2014). Plastic modulation of PTSD resting-state networks and subjective wellbeing by EEG neurofeedback. Acta Psychiatrica Scandinavica, 130(2), 123136. https://doi.org/10.1111/acps.12229

Koberda, J. L. (2015a). LORETA z-score neurofeedback: Effectiveness in rehabilitation of patients suffering from traumatic brain injury. Journal of Neurology and Neurobiology, 1(4), 1-9. https://doi.org/10.16966/2379-7150.113

Koberda, L. (2012). Autistic spectrum disorder as a potential target of z-score LORETA neurofeedback. NeuroConnections, Winter 2012, 24-25.

Koberda, L. (2014a). Z-score LORETA neurofeedback as a potential rehabilitation modality in patients with CVA. Journal of Neurology \& Stroke, 1(5), 1-5. https://doi.org/10.15406 /jnsk.2014.01.00029

Koberda, L. (2014b). Z-score LORETA neurofeedback as a potential therapy in cognitive dysfunction and dementia. Journal of Psychology \& Clinical Psychiatry, 1(6), 00037. https://doi.org/10.15406/jpcpy.2014.01.00037

Koberda, J. L. (2015b). Z-score LORETA neurofeedback as a potential therapy for patients with seizures and refractory epilepsy. Journal of Neurology and Neurobiology, 1(1), 1-6. https://doi.org/10.16966/2379-7150.101

Koberda, J. L., Koberda, P., Moses, A., Winslow, J., Bienkiewicz, A., \& Koberda, L. (2014a). Z-score LORETA neurofeedback as a potential therapy for ADHD. Biofeedback, 42(2), 74-81. https://doi.org/10.5298/1081-5937-42.2.05

Koberda, L., Koberda, P., Moses, A., Winslow, J., Bienkiewicz, A., \& Koberda, L. (2014b). Z-score neurofeedback as a potential therapy in depression and anxiety. NeuroConnections, Spring 2014, 52-55.

Krigbaum, G., \& Wigton, N. L. (2014). When discussing neurofeedback, does modality matter? NeuroRegulation, 1(1), 48. https://doi.org/10.15540/nr.1.1.48

Kubios HRV analysis software (version 2.1) [Computer software]. (n.d.). Kuopio, Finland: Biosignal Analysis and Medical Imaging Group.

Lambos, W. A., \& Williams, R. A. (2015a). Treating anxiety disorders using z-scored EEG neurofeedback. In R. W. Thatcher \& J. F. Lubar (Eds.), Z score neurofeedback: Clinical applications (pp. 189-217). San Diego, CA: Academic Press. https://doi.org/10.1016/B978-0-12-801291-8.00009-1

Lambos, W. A., \& Williams, R. A. (2015b). Treating executive functioning disorders using LORETA z-scored EEG biofeedback. In R. W. Thatcher \& J. F. Lubar (Eds.), Z score neurofeedback: Clinical applications (pp. 141-157). San Diego, CA: Academic Press. https://doi.org/10.1016/B978-012-801291-8.00007-8

La Vaque, T. J., Hammond, D. C., Trudeau, D., Monastra, V., Perry, J., Lehrer, P., ... Sherman, R. (2002). Template for developing guidelines for the evaluation of the clinical efficacy of psychophysiological interventions. Applied Psychophysiology and Biofeedback, 27(4), 273-281. https://doi.org/10.1023/A:1021061318355

Lanius, R. A., Frewen, P. A., Tursich, M., Jetly, R., \& McKinnon, M. C. (2015). Restoring large-scale brain networks in PTSD and related disorders: A proposal for neuroscientifically-informed treatment interventions. European Journal of Psychotraumatology, 6(1), 27313. https://doi.org/10.3402 lejpt.v6.27313

Lehrer, P. M., Vaschillo, E., \& Vaschillo, B. (2000). Resonant frequency biofeedback training to increase cardiac variability: Rationale and manual for training. Applied Psychophysiology and Biofeedback, 25(3), 177-191. https://doi.org/10.1023 /A:1009554825745

Leon, A. C., Davis, L. L., \& Kraemer, H. C. (2011). The role and interpretation of pilot studies in clinical research. Journal of Psychiatric Research, 45(5), 626-629. https://doi.org/10.1016 jj.jpsychires.2010.10.008
LORETA Current Density Normative Database [Computer software]. (n.d.). St. Petersburg, FL: Applied Neuroscience, Inc. Retrieved from https://appliedneuroscience.com/product /loreta-current-density-normative-db/

Markiewicz, R. (2017). The use of EEG Biofeedback /Neurofeedback in psychiatric rehabilitation. Psychiatria Polska, 51(6), 1095-1106. https://doi.org/10.12740/PP/68919

McCraty, R., \& Shaffer, F. (2015). Heart rate variability: New perspectives on physiological mechanisms, assessment of self-regulatory capacity, and health risk. Global Advances in Health and Medicine, 4(1), 46-61. https://doi.org/10.7453 /gahmj.2014.073

Menon, V. (2011). Large-scale brain networks and psychopathology: A unifying triple network model. Trends in Cognitive Sciences, 15(10), 483-506. https://doi.org/10.1016 /j.tics.2011.08.003

Najavits, L. M. (2015). The problem of dropout from "gold standard" PTSD therapies. F1000Prime Reports, 7, 43. https://doi.org /10.12703/P7-43

NeuroGuide (Version 2.9.1) [Computer software]. (n.d.). Largo, FL: Applied Neuroscience, Inc.

Othmer, S. (2012). Psychological health and neurofeedback: Remediating PTSD and TBI. Woodland Hills, CA: The EEG Institute. Retrieved from http://www.eeginfo-europe.com /fileadmin/images/research/anxiety/RemediatingPTSD_TBI.p df

Panisch, L. S., \& Hai, A. H. (2018). The effectiveness of using neurofeedback in the treatment of post-traumatic stress disorder: A systematic review. Trauma, Violence \& Abuse. https://doi.org/10.1177/1524838018781103

Paret, C., Kluetsch, R., Ruf, M., Demirakca, T., Hoesterey, S., Ende, G., \& Schmahl, C. (2014). Down-regulation of amygdala activation with real-time fMRI neurofeedback in a healthy female sample. Frontiers in Behavioral Neuroscience, 8, 299. https://doi.org/10.3389/fnbeh.2014.00299

Pascual-Marqui, R. D., Esslen, M., Kochi, K., \& Lehmann, D. (2002). Functional imaging with low-resolution brain electromagnetic tomography (LORETA): A review. Methods and Findings in Experimental and Clinical Pharmacology, 24(Suppl. C), 91-95.

Pascual-Marqui, R. D., Michel, C. M., \& Lehmann, D. (1994). Low resolution electromagnetic tomography: A new method for localizing electrical activity in the brain. International Journal of Psychophysiology, 18(1), 49-65. https://doi.org/10.1016 10167-8760(84)90014-X

Patel, R., Spreng, R. N., Shin, L. M., \& Girard, T. A. (2012). Neurocircuitry models of posttraumatic stress disorder and beyond: A meta-analysis of functional neuroimaging studies. Neuroscience \& Biobehavioral Reviews, 36(9), 2130-2142. https://doi.org/10.1016/j.neubiorev.2012.06.003

Peniston, E. G., \& Kulkosky, P. J. (1991). Alpha-theta brainwave neuro-feedback therapy for Vietnam veterans with combatrelated post-traumatic stress disorder. Medical Psychotherapy, 4, 47-60.

Peniston, E. G., Marrinan, D. A., Deming, W. A., \& Kulkosky, P. J. (1993). EEG alpha-theta brainwave synchronization in Vietnam theater veterans with combat-related post-traumatic stress disorder and alcohol abuse. Advances in Medical Psychotherapy, 6, 37-50.

Pop-Jordanova, N., \& Zorcec, T. (2004). Child trauma, attachment and biofeedback mitigation. Prilozi / Makedonska Akademija Na Naukite I Umetnostite, Oddelenie Za Biološki I Medicinski Nauki = Contributions / Macedonian Academy of Sciences and Arts, Section of Biological and Medical Sciences, 25(1-2), 103-114.

Reiter, K., Andersen, S. B., \& Carlsson, J. (2016). Neurofeedback treatment and posttraumatic stress disorder: Effectiveness of neurofeedback on posttraumatic stress disorder and the optimal choice of protocol. The Journal of Nervous and Mental 
Disease, 204(2), 69-77. https://doi.org/10.1097 /NMD.0000000000000418

Reyes, F. J. (2014). Implementing heart rate variability biofeedback groups for veterans with posttraumatic stress disorder. Biofeedback, 42(4), 137-142. https://doi.org/10.5298/10815937-42.4.02

Ros, T., Baars, B. J., Lanius, R. A., \& Vuilleumier, P. (2014). Tuning pathological brain oscillations with neurofeedback: A systems neuroscience framework. Frontiers in Human Neuroscience, 8, 1008. https://doi.org/ 10.3389/fnhum.2014.01008

Shaffer, F., \& Combatalade, D. C. (2013). Don't add or miss a beat: A guide to cleaner heart rate variability recordings. Biofeedback, 41(3), 121-130. https://doi.org/10.5298/10815937-41.3.04

Simmons, A. N., Paulus, M. P., Thorp, S. R., Matthews, S. C., Norman, S. B., \& Stein, M. B. (2008). Functional activation and neural networks in women with posttraumatic stress disorder related to intimate partner violence. Biological Psychiatry, 64(8), 681-690. $\quad$ https://doi.org/10.1016 /j.biopsych.2008.05.027

Simmons, A., Strigo, I. A., Matthews, S. C., Paulus, M. P., \& Stein, M. B. (2009). Initial evidence of a failure to activate right anterior insula during affective set shifting in posttraumatic stress disorder. Psychosomatic Medicine, 71(4), 373-377. https://doi.org/10.1097/PSY.0b013e3181a56ed8

Smith, W. D. (2008). The effect of neurofeedback training on PTSD symptoms of depression and attention problems among military veterans (Doctoral dissertation, Capella University). Retrieved from http://gradworks.umi.com/33/15/3315214.html

Strehl, U. (2014). What learning theories can teach us in designing neurofeedback treatments. Frontiers in Human Neuroscience, 8, 894. https://doi.org/10.3389/fnhum.2014.00894

Swift, J. K., \& Greenberg, R. P. (2012). Premature discontinuation in adult psychotherapy: A meta-analysis. Journal of Consulting and Clinical Psychology, 80(4), 547-559. https://doi.org /10.1037/a0028226

Tan, G., Dao, T. K., Farmer, L., Sutherland, R. J., \& Gevirtz, R. (2011). Heart rate variability (HRV) and posttraumatic stress disorder (PTSD): A pilot study. Applied Psychophysiology and Biofeedback, 36(1), 27-35. https://doi.org/10.1007/s10484010-9141-y

Thatcher, R. W. (2010). Validity and reliability of quantitative electroencephalography (qEEG). Journal of Neurotherapy, 14(2), 122-152. https://doi.org/10.1080/10874201003773500

Thatcher, R. W., \& Lubar, J. F. (2009). History of the scientific standards of QEEG normative databases. In T. H. Budzinsky, H. K. Budzinsky, J. R. Evans, \& A. Abarbanel (Eds.), Introduction to Quantitative EEG and neurofeedback: Advanced theory and applications (2nd ed., pp. 29-59). San Diego, CA: Academic Press.

Thatcher, R. W. (2013). Latest developments in live z-score training: Symptom check list, phase reset, and LORETA zscore biofeedback. Journal of Neurotherapy, 17(1), 69-87. https://doi.org/10.1080/10874208.2013.759032

Thatcher, R. W., North, D., \& Biver, C. (2005). Evaluation and validity of a LORETA normative EEG database. Clinical EEG and Neuroscience, 36(2), 116-122. https://doi.org/ $10.1177 / 155005940503600211$

Thatcher, R. W., Walker, R. A., Biver, C. J., North, D. N., \& Curtin, R. (2003). Quantitative EEG normative databases: Validation and clinical correlation. Journal of Neurotherapy, 7(3-4): 87121. https://doi.org/10.1300/J184v07n03_05

Thayer, J. F., Ahs, F., Fredrikson, M., Sollers, J. J., \& Wager, T. D. (2012). A meta-analysis of heart rate variability and neuroimaging studies: Implications for heart rate variability as a marker of stress and health. Neuroscience \& Biobehavioral Reviews, 36(2), 747-756. https://doi.org/10.1016 /j.neubiorev.2011.11.009

Thompson, M., Thompson, L., \& Reid-Chung, A. (2015). Treating postconcussion syndrome with LORETA $z$-score neurofeedback and heart rate variability biofeedback: Neuroanatomical/neurophysiological rationale, methods, and case examples. Biofeedback, 43(1), 15-26. https://doi.org/ 10.5298/1081-5937-43.1.07

Todder, D., Levine, J., Abujumah, A., Mater, M., Cohen, H., \& Kaplan, Z. (2012). The quantitative electroencephalogram and the low-resolution electrical tomographic analysis in posttraumatic stress disorder. Clinical EEG and Neuroscience, 43(1), 48-53. https://doi.org/10.1177/1550059411428716

van Boxtel, G. J. M., Denissen, A. J. M., Jäger, M., Vernon, D., Dekker, M. K. J., Mihajlović, V., \& Sitskoorn, M. M. (2012). A novel self-guided approach to alpha activity training. International Journal of Psychophysiology, 83(3), 282-294. https://doi.org/10.1016/j.ijpsycho.2011.11.004

van der Kolk, B. A. (2006). Clinical implications of neuroscience research in PTSD. Annals of the New York Academy of Sciences, 1071(1), 277-293. https://doi.org/10.1196 lannals.1364.022

van der Kolk, B. A., Hodgdon, H., Gapen, M., Musicaro, R., Suvak, M. K., Hamlin, E., \& Spinazzola, J. (2016). A randomized controlled study of neurofeedback for chronic PTSD. PLOS ONE, 11(12), e0166752. https://doi.org/10.1371 /journal.pone.0166752

Wahbeh, H., \& Oken, B. S. (2013). Peak high-frequency HRV and peak alpha frequency higher in PTSD. Applied Psychophysiology and Biofeedback, 38(1), 57-69. https://doi.org/10.1007/s10484-012-9208-z

Walker, J. E. (2009). Anxiety associated with post traumatic stress disorder-the role of quantitative electroencephalograph in diagnosis and in guiding neurofeedback training to remediate the anxiety. Biofeedback, 37(2), 67-70. https://doi.org /10.5298/1081-5937-37.2.67

Watts, B. V., Shiner, B., Zubkoff, L., Carpenter-Song, E., Ronconi, J. M., \& Coldwell, C. M. (2014). Implementation of evidencebased psychotherapies for posttraumatic stress disorder in VA specialty clinics. Psychiatric Services, 65(5), 648-653. https://doi.org/10.1176/appi.ps.201300176

Weaver, A., \& Goldberg, S. (2012). Clinical biostatistics and epidemiology made ridiculously simple (1st ed.). Miami, FL: MedMaster, Inc.

World Medical Association. (2013). WMA Declaration of Helsinki Ethical principles for medical research involving human subjects. Retrieved August 7, 2017, from https://www.wma.net/policies-post/wma-declaration-ofhelsinki-ethical-principles-for-medical-research-involvinghuman-subjects/

Zucker, T. L., Samuelson, K. W., Muench, F., Greenberg, M. A., \& Gevirtz, R. N. (2009). The effects of respiratory sinus arrhythmia biofeedback on heart rate variability and posttraumatic stress disorder symptoms: A pilot study. Applied Psychophysiology and Biofeedback, 34(2), 135-143. https://doi.org/10.1007/s10484-009-9085-2

Zukor Media Player [Computer software]. (n.d.). Las Vegas, NV: Zukor Interactive.

Received: April 30, 2019

Accepted: May 17, 2019

Published: June 26, 2019 